\title{
Application of Coincidence Doppler Broadening Spectroscopy to Hydrocarbons at Different Substance States
}

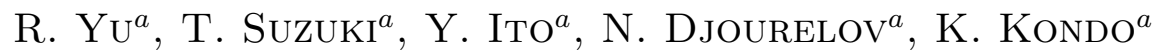 \\ AND V.P. SHANTAROVICH ${ }^{a, b}$ \\ ${ }^{a}$ Radiation Science Center \\ High Energy Accelerator Research Organization (KEK) \\ 1-1 Oho, Tsukuba, Ibaraki 305-0801, Japan \\ ${ }^{b}$ Semenov-Institute of Chemical Physics, Russian Academy of Sciences \\ GSP-1, Kosygin st. 4, Moscow 119977, Russia
}

\begin{abstract}
Coincidence Doppler broadening spectroscopy was applied to study $n$-hexane $\left(\mathrm{C}_{6} \mathrm{H}_{14}\right)$, cyclohexane $\left(\mathrm{C}_{6} \mathrm{H}_{12}\right)$ and cyclohexanone $\left(\mathrm{C}_{6} \mathrm{H}_{10} \mathrm{O}\right)$, at solid, as well as liquid states. The experiments were performed in parallel with traditional positron annihilation lifetime spectroscopy. Significant positron trapping by oxygen was observed in cyclohexanone; the oxygen effect is stronger in liquid state than in solid one, which can possibly be attributed to positron solvation in polar solution. Coincidence Doppler broadening results for $n$-hexane and cyclohexane indicate an existence of higher energetic annihilation electrons in Ps-bubble forming liquids than that in solids.
\end{abstract}

PACS numbers: 36.10.Dr, 82.30.Gg, 78.70.Bj

\section{Introduction}

Positron annihilation lifetime (PAL) and coincidence Doppler broadening (CDB) [1] measurements are often carried out simultaneously for better understanding of the annihilation processes in matter. In the present work, we studied simple hydrocarbon samples, $n$-hexane $\left(\mathrm{C}_{6} \mathrm{H}_{14}\right)$, cyclohexane $\left(\mathrm{C}_{6} \mathrm{H}_{12}\right)$ and cyclohexanone $\left(\mathrm{C}_{6} \mathrm{H}_{10} \mathrm{O}\right)$, at solid and liquid states using these two techniques. Our aim is not only to examine the correlation between constitute atoms and the high 
momentum region in measured CDB spectra [2], but also try to investigate the effect of unique phenomena in liquids [3] such as Ps bubble formation and positron solvation on the momentum distribution of annihilation electrons.

\section{Experimental}

A $1.1 \mathrm{MBq}$ positron source, ${ }^{22} \mathrm{NaCl}$ enveloped by kapton foils, were used. The source was put into one arm of a breeches glass tube having a vacuum stopcock. The sample liquid was introduced into the other arm, where freeze-pump-thaw was carried out for degasification. Measurements in solid states were realized by freezing the liquids using dry ice $\left(-78^{\circ} \mathrm{C}\right)$.

PAL spectra were recorded with a conventional fast-fast coincidence system having a time resolution of about $300 \mathrm{ps}$ FWHM. All the spectra were resolved into three components using PATFIT code without source correction; the longest-lived component with a lifetime $\tau_{3}$ and intensity $I_{3}$ was attributed to the pick-off annihilation of $o$-Ps atoms. CDB measurements were simultaneously carried out with two HPGe detectors set face to face to detect coincidences of two $0.511 \mathrm{MeV}$ annihilation photons. Total resolution of this system is about $1.0 \mathrm{keV}$ FWHM. The CDB data were read out at every $4 \mathrm{~h}$, resulting in a total counts at least $\approx 2.8 \times 10^{6}$ in the $256 \times 256$ coincidence matrix and $\approx 0.9 \times 10^{6}$ in the extracted coincidence diagonal cut.

\section{Results and discussions}

Figure 1 shows PALS results for cyclohexane measured at its solid and liquid states. Evidently, o-Ps pick-off annihilation resulted in significantly larger $\tau_{3}$ and $I_{3}$ in liquid state. Similar change was also observed for $n$-hexane, the results showed that $\tau_{3}$ for its solid state is about $3.2 \mathrm{~ns}$, then increased to $4.0 \mathrm{~ns}$ for liquid state. Meanwhile, $I_{3}$ was raised from $22.5 \%$ to $32.5 \%$. In contrast, one cannot observe clear change in $I_{3}(14.2 \%)$ between liquid and solid cyclohexanone, despite a strong reduction of $\tau_{3}$ from $2.7 \mathrm{~ns}$ for solid to $1.2 \mathrm{~ns}$ for liquid state. The larger $o$-Ps lifetimes observed at liquid state can be understood as due to the formation of Ps bubbles. As is well known, the larger the bubble/cavity size the longer the $o$-Ps atom is alive [4]. In the solid, o-Ps may be confined in already existing holes or cavities, which seems smaller than the $o$-Ps bubbles in liquids as indicated from the current lifetime results. Unlike $\tau_{3}$, the Ps formation probability is usually a more complicated case. According to the spur model [3], Ps is formed by a reaction between the positron and one of the electrons in the terminal positron spur when the energetic positron loses the last part of its kinetic energy in a material. This process competes with the recombination of electrons and positive ions, out-diffusion of positrons and electrons from the spurs, reactions of the positrons and the electrons with the solvent molecules or with scavengers in 


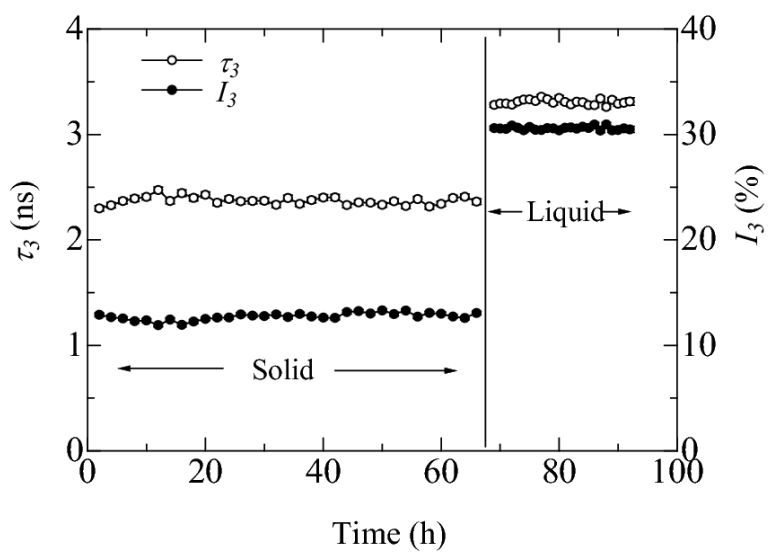

Fig. 1. o-Ps annihilation lifetime $\left(\tau_{3}\right)$ and intensity $\left(I_{3}\right)$ for cyclohexane at solid, as well as liquid state.

the spur, etc. In the present case, Ps formation probability may be closely related to the polar and nonpolar characteristics of the studied samples. Cyclohexanone belongs to polar molecules because of the constituent oxygen atom and dipolar character of $\mathrm{C}_{6}^{+}=\mathrm{O}^{-}$. It was reported that in polar liquids, reduction of Ps formation probability often happens due to electron and positron solvation as well as dielectric shielding effect of the positron and electrons [5]. In contrast, dipolar effect is somewhat weakened in solid states because of the possible existence of some well-ordered polar molecules. This makes the difference between $I_{3}$ for liquid and solid cyclohexanone almost unnoticeable.

The above-mentioned positron solvation has often been suggested, but without direct evidence so far. This probably has been shown for the first time in liquid cyclohexanone by the present CDB measurements. Figure 2 shows the ratio curves

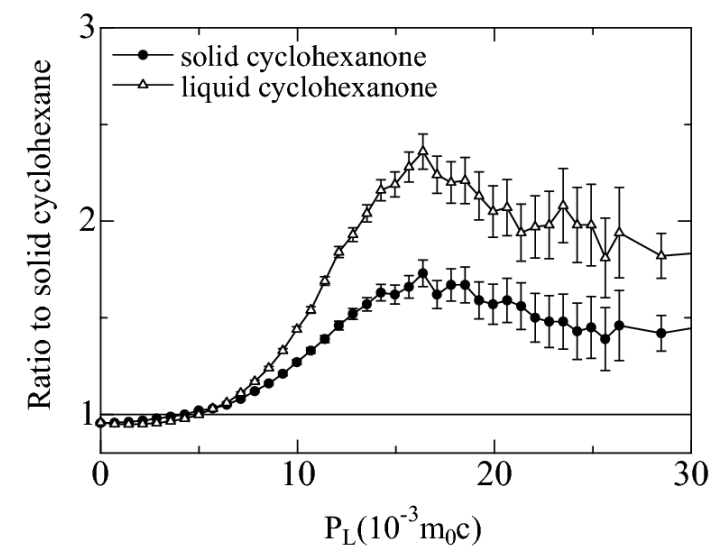

Fig. 2. Ratio curves of CDB spectra for solid and liquid cyclohexanone relative to solid cyclohexane. $P_{\mathrm{L}}$ is the momentum component of the positron-electron annihilation pair in the direction of the detectors. 
of CDB spectra for solid and liquid cyclohexanone with respect to solid cyclohexane. The prominent peak at around $16 \times 10^{-3} m_{0} c$ is the fingerprint of positrons annihilation with high-momentum electrons belonging to oxygen [6]. In addition, one can find that the peak for liquid state is stronger than that for solid. Considering the same positronium formation probability in the two substance states, fraction of free positrons escaping Ps formation, $f$, should also be the same between them in the light of the relationship $f=1-\frac{4}{3} I_{3}$. The enhanced oxygen peak in the $\mathrm{CDB}$ ratio curve for liquid cyclohexanone indicates that considerable amount of free positrons, most probably solvated positrons, were surrounded solely by oxygen atoms and finally annihilate on them. For solid case, there must be a larger probability for free positrons to annihilate with valence electrons in the $\mathrm{C}-$ $\mathrm{C}$ and $\mathrm{C}-\mathrm{H}$ bonds, which is known to result in a momentum distribution with relatively smaller $P_{\mathrm{L}}$ values $[7]$.

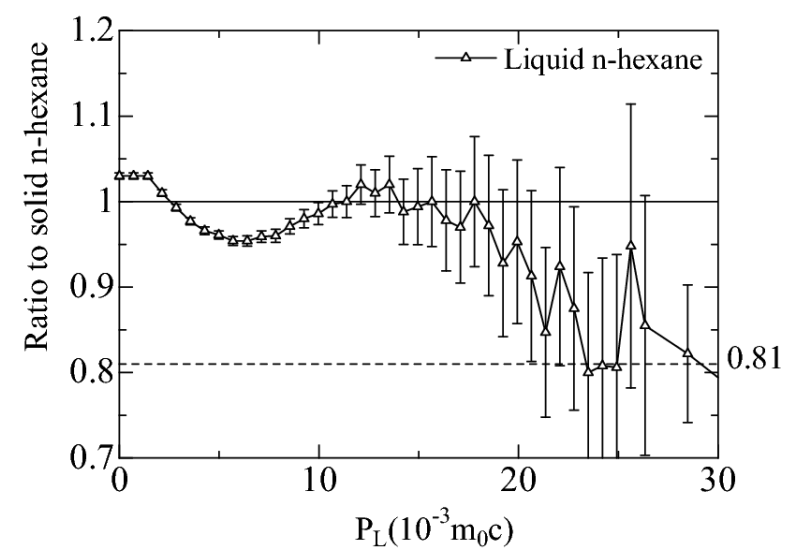

Fig. 3. Ratio curve of CDB spectra for liquid $n$-hexane relative to its solid state. The dashed line corresponds to the ratio between free positron annihilation fractions in the two samples.

Returning to the observed larger Ps formation probabilities for liquid $n$-hexane and cyclohexane compared with their corresponding solid states, which can partially be attributed to the formation of Ps bubbles in liquids. It was observed that Ps bubble formation also significantly affects the measured CDB spectra. As shown in Fig. 3, the ratio curve for liquid $n$-hexane relative to its solid state is significantly enhanced in the momentum region from about 5 to $20 \times 10^{-3} m_{0} c$, since the expected ratio [8] due to $I_{3}$ (or $f$ as mentioned above) difference is only about 0.81 . It might indicate that electrons, which annihilate with positrons via pick-off process in the bubble-forming liquids, maintain larger energies than that in solids. The reason accounting for this experimental result is under consideration. 


\section{Summaries}

$o$-Ps lifetime and intensity for nonpolar $n$-hexane and cyclohexane increase from solid to liquid state. The lifetime enhancement was attributed to the Ps bubble formation. For liquid $n$-hexane and cyclohexane, Ps bubble formation leads to a wider Doppler broadening curve than that of solids. Solvation of positrons in liquid cyclohexanone enhances the oxygen-specific peak in its CDB ratio curve, and reduces the probability for free positrons to be annihilated with electrons belonging to $\mathrm{C}-\mathrm{C}$ and $\mathrm{C}-\mathrm{H}$ bonds.

\section{Acknowledgments}

This work was supported by a Grant-in-Aid in Scientific Research by the Japanese Ministry of Education, Culture, Sports, Science and Technology. R.Y. would like to thank Prof. T. Shibata and Dr. K. Bessho for their help to conduct research at KEK. Valuable discussion with Dr. Yutaka Ito is deeply appreciated.

\section{References}

[1] K.G. Lynn, J.R. MacDonald, R.A. Boie, L.C. Feldman, J.D. Gabbe, M.F. Robbins, E. Bonderup, J. Golovchenko, Phys. Rev. Lett. 38, 241 (1977).

[2] P. Asoka-Kumar, M. Alatalo, V.J. Ghosh, A.C. Kruseman, B. Nielsen, K.G. Lynn, Phys. Rev. Lett. 77, 2097 (1996).

[3] E. Mogensen, Positron Annihilation in Chemistry, Springer-Verlag, Berlin 1995, p. 53 .

[4] S.J. Tao, J. Chem. Phys. 56, 5499 (1972).

[5] S.Y. Chuang, Chin. J. Phys. 16, 111 (1978).

[6] Y. Nagai, M. Hasegawa, Y. Kobayashi, C.L. Wang, W. Zheng, C. Zhang, Phys. Rev. B 60, 11863 (1999).

[7] S.Y. Chuang, B.G. Hogg, Can. J. Phys. 45, 3895 (1967).

[8] N. Djourelov, C. He, T. Suzuki, V. Shantarovich, Y. Ito, K. Kondo, Y. Ito, Chem. Phys. 302, 179 (2004). 